\title{
Determination of the Preliminary Phase of the Facility Destruction Based on the Resistance- Acoustic Method of Control
}

\author{
Oleg R. Kuzichkin ${ }^{1}$, Artem A. Bykov ${ }^{2}$, Nikolay V. Dorofeev ${ }^{2}$, Konstantin V. Podmasteriev ${ }^{3}$ \\ ${ }^{1}$ Belgorod National Research University, 85 Pobedy st., Belgorod 308015, 1155464@bsu.edu.ru \\ ${ }^{2}$ Vladimir State University named after A. G. and N. G. Stoletovs, 23 Orlovskaya st., Murom 602264, itpu@mivlgu.ru \\ ${ }^{3}$ Orel State Technical University, 29 Naugorskoe highway, Orel 302020, wladimir@orel.ru
}

\begin{abstract}
The article substantiates the use of the resistance-acoustic method in problems of non-destructive testing of mechanical stresses or defects in structures of metals and alloys in natural and technical systems. The possibilities of the use of the proposed method for the potential monitored facilities were analyzed, to which the strict requirements for operational security are imposed (bearing reinforced concrete structures, bridges, radio and television towers, etc.). The main approaches to the development of the application methodology of the resistance-acoustic effect in solving the problems of technological control of mechanical stresses and defects in structures of metals and alloys are determined. The mathematical modeling was performed, using basic mathematical models of facilities of the resistance-acoustic control, considering the mutual influence of the elastic and electric fields in electrically conductive media, taking into consideration the parameters of technical state and wear.
\end{abstract}

Keywords-resistance-acoustic control, measuring system, natural-technical systems, non-destructive testing, metal constructions

\section{INTRODUCTION}

The problem of ensuring the operational reliability of technical objects and creation of effective technical control systems of the state of bearing metal structures is an important area of modern engineering. During longterm operation, the metal structures of technical objects are exposed to constant mechanical, physical and chemical effects, which result in their physical wear and impairment of operating parameters of the facilities [1, 2]. Studies show [3, 4] that an important reason for the decline in performance and durability of steel structures are climatic effects. Treat the main reasons exerting negative impact on steel structures: humidity, temperature, formation of condensate, dust, aggressive gases, liquids and technological emissions at manufacturing enterprises, and the power loadings characteristic of different types of industrial activity. The consequence of natural disasters can be an accident, as a sudden failure of the structure [5].
Technogenic factors can cause direct destruction of designs because of accidents or inadmissible loading. Over time, there are also processes of accumulation and development of damages, aging of materials.

For bridges, these are loads from vehicles that affect construction or traffic accidents, which can cause direct destruction of the structure or unacceptable load for the bridge, which eventually leads to the accumulation and development of damages and premature aging of materials. For pipelines, these are hydraulic shocks [6].

Under the influence of factors of both groups, there can be damages of designs in the form of a deviation of their geometrical parameters from initial, and in the form of destructions of designs. All this is the reason of development of initial defects of a design.

Serious accidental consequences can lead to hidden processes of local corrosion of concrete and reinforcement, not detected in a timely manner. These destructive processes are also aggravated with shortcomings of the constructive technology solutions put in the project. Complicating the overall environmental situation has a growing destabilizing effect on engineering structures. In such conditions, even full compliance with the norms of operation of the structure is not a guarantee of a sustainable implementation of the regulatory operating cycle of the facility [7].

There are cases when the wear processes of metal structures pass almost imperceptibly until the appearance of irreversible processes and their catastrophic destruction [8]. The performance of their constant monitoring is necessary in order to control their technical condition, for operational control and the development of measures to restore the operational qualities of the structures.

One of the promising fields of modern defect detection is the use of resistance methods of nondestructive testing, which are based on the dependence of the electrical resistance or conductivity of the elements of metal structures on the operating strains in them [9]. But these methods do not allow localizing the location of defects. This is especially true for long products, in which 
access to the entire surface is difficult or impossible. Recently, however, due to increasing demands to operational security of industrial facilities, there is a necessity to develop new more effective control methods that enable to implement early detection of preemergency status of metal structures on the test facility. This problem is especially relevant for industrial and social facilities of increased risk.

One of the possible approaches to solve this problem is the simultaneous use of resistance and acoustic methods $[10,11]$. At that, the recorded parameter is the electric resistance of the element of the controlled metal structure, carrying the information about mutual effects of electrical and acoustic transformations and respectively on the technological state of the element.

Mechanical stresses in metals depend directly and essentially on temperature impacts, including temperature oscillations. In that connection, the recent climate changes can consider as additional factors of risk, because of latent qualitative changes in local climate dynamics can introduce essential corrections in economic estimations of climate-related damages [12]. In particular, variable patterns of space heterogeneities of annual warming-cooling cycles [13] and abrupt temperature shifts within interseasonal hysteresis regularities [14] are typical for Vladimir region, where our experimental tests are made.

The principal possibility of technological control of metal structures based on the resistance-acoustic method is determined by the fact that in case of elastic impact on the conductive element, its electrical resistance is changed due to piezoresistance and tensoresistance sensitivity, as well as due to dependence of magnetic permeability on the mechanical stresses in it [15].

The aim of this work is the substantiation of application of the resistance-acoustic method in problems of non-destructive testing of mechanical stresses and defects in metal and alloy structures in natural and technical systems.

\section{THEORETICAL BASIS OF THE APPLICATION OF THE RESISTANCE-ACOUSTIC CONTROL METHOD}

The principle of the application of the resistanceacoustic method of defect detection is based on the correlation between the integrated electrical and mechanical properties of metals and alloys, which are electrical conductivity or electrical resistivity in the $h$ layer of metal and the deforming ability of residual stresses, associated with changes in the crystal lattice during deformation [16].

There is a relation between the electrical conductivity $\gamma$ and mechanical stresses in metals is determined by formula [17]:

$$
\gamma=\frac{e^{2} E n_{0}}{m k T V_{T} N_{0} \pi} \cdot d
$$

where $e-$ is the electron charge; $m$-is the mass of the electron rest; $n_{0}-$ is the number of conduction electrons per unit volume; $E-$ is the field strength; $k-$ is Boltzmann constant; $T$ - is the absolute temperature in degrees Kelvin; $V_{T}$ - is thermal velocity of the electrons; $N_{0}$-is the number of atoms per unit volume; $d-$ is the period of the crystal lattice.

The dependence of conductivity $\gamma$ from an electrical resistivity: $\rho=1 / \gamma$.

In the absence of mechanical stresses, metal has a nominal value of the lattice period $d_{0}$ and the corresponding nominal value of electrical conductivity $\gamma_{0}$. Under the influence of changes in mechanical stresses $\Delta \sigma$, a change in the metal lattice period $\Delta d$ occurs. In the elastic deformation zone, this change can be considered linear and proportional to the mechanical stress. In accordance with (1), a change in the electrical conductivity $\Delta \gamma$ is also proportional to average mechanical stresses $\Delta \sigma$ by the formula:

$$
\Delta \gamma=K_{\sigma} \Delta \sigma
$$

where $K_{\sigma}$-is coefficient of the material properties.

Thus, by measuring the electrical properties of conductive products, under the simultaneous influence of mechanical stresses the presence of signs of the initial phase of the product distraction can be diagnosed with a deviation from the relation (1). In addition, for the registration of the resistivity distribution in depth the phenomenon of skin effect is used, in which the highfrequency currents are concentrated at such surface of the conductor which is the closest to the field sources causing the occurrence of currents [17]. The current penetration depth $\mathrm{h}$ in such conductor is defined by the expression:

$$
h=\frac{1}{\sqrt{\pi f \mu \gamma}}
$$

where $f-$ is the current rate; $\mu-$ is magnetic permeability of the material; $\gamma-$ is conductivity material.

In this case, based on the use of the resistanceacoustic method, it is possible to locate defects in depth due to variations in the frequency of resistance measurement. Thus, there is no need to provide access to the entire surface of an extended object of control, and by application of the skin effect, the mechanical stress distribution in the depth of the product under study is possible to obtain. 


\section{The Results of Mathematical Modeling}

Fig. 1 shows the theoretical dependence of the electrical resistance on the mechanical load applied to the test object. The theoretical graph follows the Hooke's law, under heavy load which exceeds the maximum permissible one, the resistance of the sample starts to decrease. Near the extremum point (point 2), the graph of the function begins to deviate from straight-line correlation. This point is called the elastic deformation transition point. At this point, there is the metal yield point. It indicates that the out-of-limit load was applied to the sample, which resulted in the changes in the crystal lattice, and as a result, the electrical conductivity changed. This change is already irreversible.

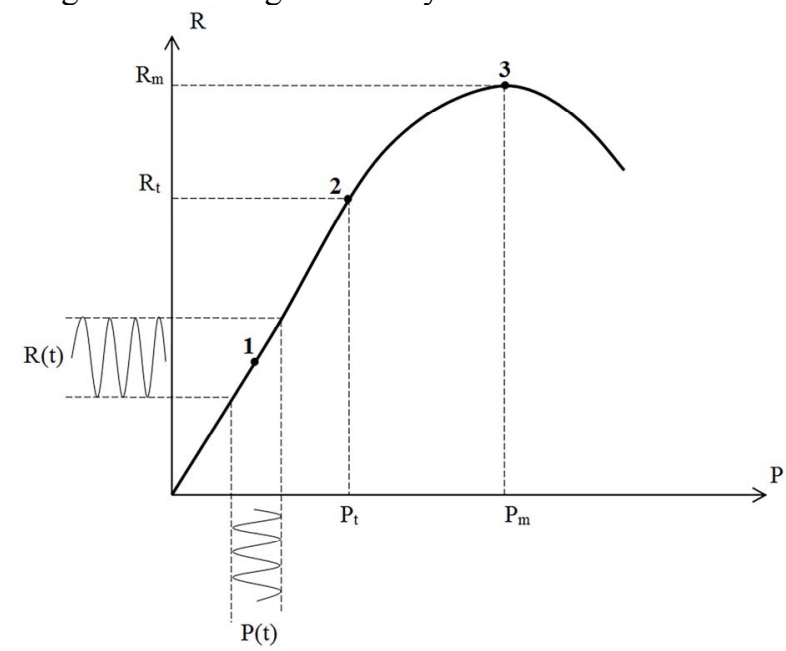

Figure 1. The theoretical dependence of the electrical resistance from the mechanical load

Obviously, when passing through this point in the registration of the spectra of simultaneously recorded electrical resistance and the applied mechanical stress they will not coincide. Accordingly, the combination frequencies will appear which can be registered by means of instrumental control.

Further, in the mathematical modeling, the theoretical dependences were obtained which showed loyalty to the theory of the dependence of the electrical resistance on the applied mechanical load on the sample under study.

$$
P(t)=P_{0}+P_{t} \cdot \sin (2 \pi \omega t)
$$

where $\omega=200 \mathrm{~Hz}$.

Theoretical dependence (Fig. 1) is approximated by the following expressions:

$$
\text { interval 1-2: } \quad R=P \frac{R_{t}}{P_{t}}
$$

interval 2-3: $\quad R=a+b P+c P^{2}$;

$$
a=R_{m}+\frac{R_{t} \cdot P_{m}^{2}}{P_{t}\left(P_{t}-P_{m}\right)} ; c=\frac{R_{t}}{2 P_{t}\left(P_{t}-P_{m}\right)} .
$$

The graph in Fig. 2 shows that spectra at input and output signals coincide in shape in point 1 . This suggests that the metal has a normal conductivity and the deformation has not yet occurred. At Fig. 3 there is a strong distortion of the spectrum in point 2 , as the metal enters the yield point. Further, in Fig. 4 (point 3) shows the appearance of side harmonics indicates the beginning of the metal destruction.

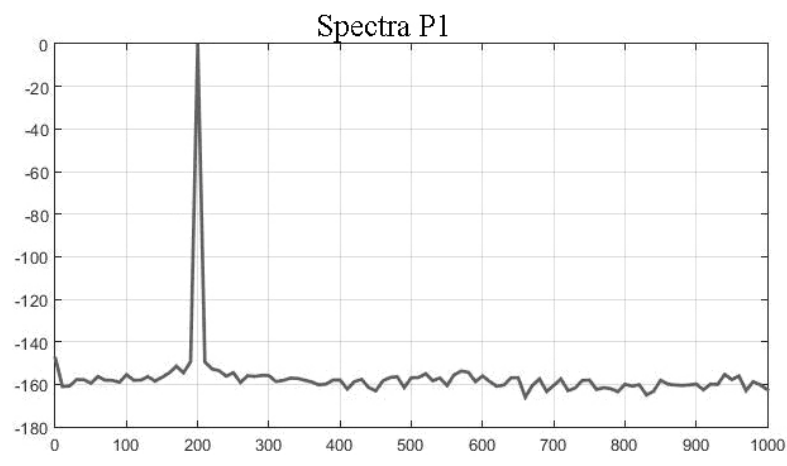

a)

Spectra R1

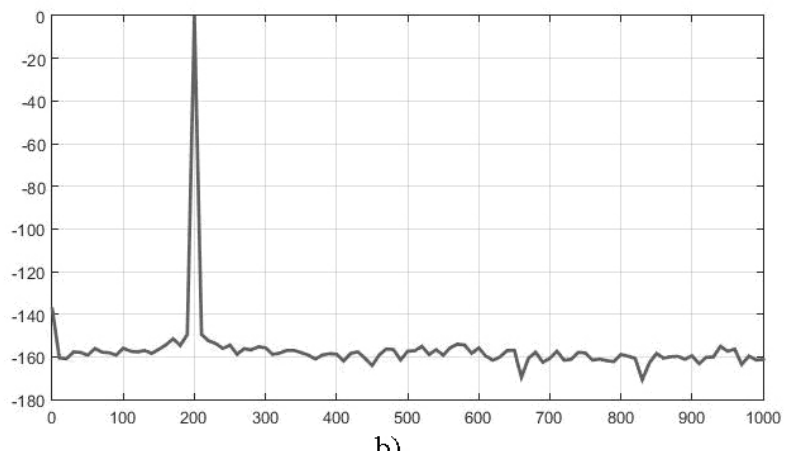

b)

Figure 2. (a) - is the signal spectrum in point 1 at the input; (b) -is the signal spectrum in point 1 at the output

Spectra P2

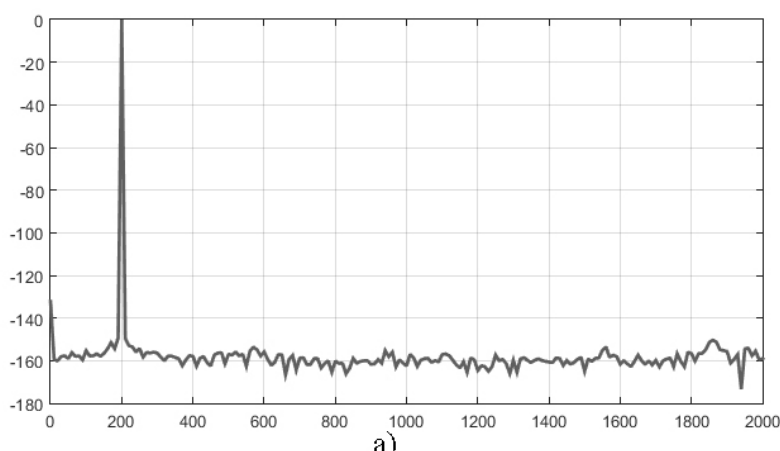


Spectra R2

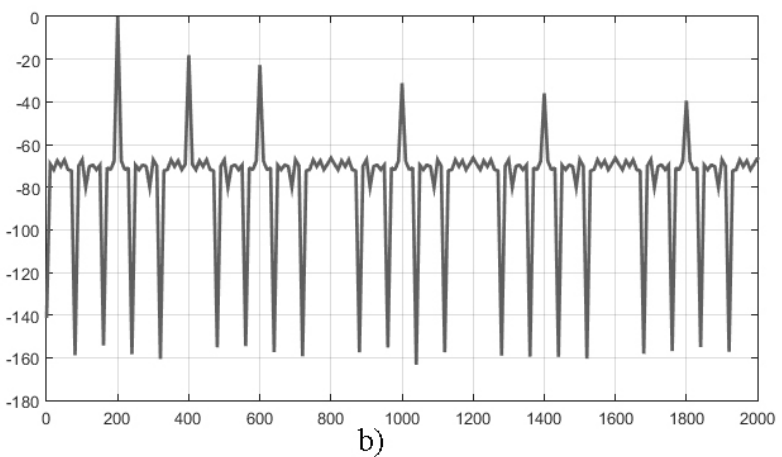

Figure 3. (a) - is the signal spectrum in point 2 at the input; (b) -is the signal spectrum in point 2 at the output

Spectra P3

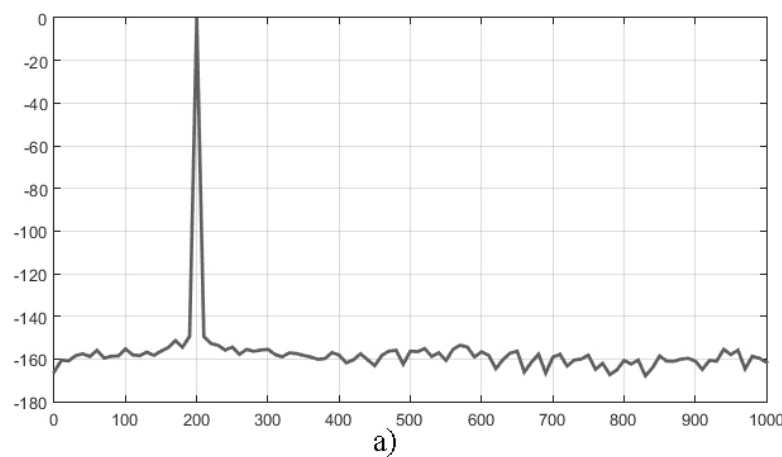

Spectra R3

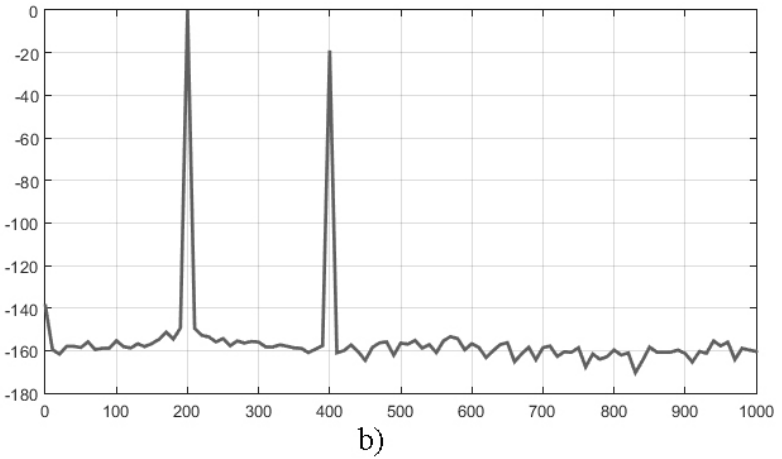

Figure 4. (a) - is the signal spectrum in point 3 at the input; (b) -is the signal spectrum in point 3 at the output

\section{CONCLUSION}

Thus, the researches conducted with application of mathematical modeling show that sharing of electric and elastic fields in problems of control for more information on a condition of a subject to control is perspective. Physically it means, that acoustic influence as if "highlights" the studied environment. Modeling has shown that the initial phase of destruction of products from metals and alloys can be found by means of electric control methods by comparison of ranges of entrance and output signals. It will allow finding the beginning of plastic deformation of a structural element before his irreversible destruction. Therefore, structural changes in the controlled environment can be found by the offered method earlier, than there will be an irreversible destruction of an object.

Application of the offered method allows to increase safety of the environment of activity of the person, to reduce risk level of emergence of technogenic accidents and accidents in natural and technical systems due to increase in reliability and efficiency of the obtained information on the current changes in a controlled metalwork, and also to define their residual resource.

\section{ACKNOWLEDGMENT}

This work was supported by a grant of the Ministry of Education and Science of the Russian Federation № 5.3606.2017/ $\mathrm{PCH}$.

\section{REFERENCES}

[1] L. Runkiewicz, "Application of non-destructive testing methods to assess properties of construction materials in building diagnostics," Architecture Civil Engineering Environment, no. 2, 2009.

[2] P. Bieder, G. Dobmann, M. Kröning, J. Liu, and J. G. Ribero, "Current NDT Research \& Development for NPP Inspections," in Proceedings of the 17th World Conference on Nondestructive Testing, 25-28 October 2008, Shanghai, China, paper 382.

[3] R. H. Jones and R. E. Ricker, "Stress-corrosion cracking materials performance and evaluation," R. H. Jones, ed., 1992, ASM International, pp. 1-40.

[4] K. R. Trethewey and J. Chamberlain, Corrosion for Science and Engineering, Longman, 1995, $466 \mathrm{p}$.

[5] B. Kühn, "Assessment of existing steel structures recommendations for estimation of the remaining fatigue life," Procedia Engineering, vol. 66, pp. 3-11, 2013.

[6] J. F. Kiefner and J. Cheryl. Trench. Oil Pipeline Characteristics and Risk Factors: Illustrations from the Decade of Construction, American Petroleum Institute, December 2001.

[7] G. C. Lee, and E. Sternberg, "A new system for preventing bridge collapses," Issues in Science and Technology, vol. 24, no. 3, pp. 31-36, 2008.

[8] R. W. Armstrong and S. M. Walley, "High strain rate properties of metals and alloys," International Materials Reviews, vol. 53, no. 3, pp. 105-128, 2008.

[9] A. Ulybin, A. Lanko, N. Vatin and K. Lysnytska, "Examination of the basement of historic buildings in investment activity," MATEC Web of Conferences, vol. 73, pp. 01007, 2016.

[10] A. A. Bykov and O. R. Kuzichkin, "Application of seismoelectric method for inspection conductive media," Applied Mechanic and Materials, vol. 490-491, pp. 1712-1716, 2013.

[11] A. A. Bykov and O. R. Kuzichkin, "Seismoelectric control of geodynamic processes in natural-technical systems," in Proceedings of the IEEE 7th International Conference on Intelligent Data Acquisition and Advanced Computing Systems (IDAACS'2013), Berlin, Germany, September 12-14, 2013, vol. 2, pp. 840-843.

[12] Yu. Kolokolov and A. Monovskaya, "Guess-work and reasonings on centennial evolution of surface air temperature in Russia. Part IV: Towards economic estimations of climate-related damages from the bifurcation analysis viewpoint?" Int. J. of Bifurcation and Chaos, vol. 26, no. 12, 1630033, 2016

[13] Yu. Kolokolov and A. Monovskaya, "Multidimensional analysis of dynamics of annual warming-cooling cycles on the basis of 
index model of temperature observations," in Proceedings of the $8^{\text {th }}$ IEEE International Conference on Intelligent Data Acquisition and Advanced Computing Systems: Technology and Applications (IDAACS'2015), Warsaw, Poland, 24-26 September 2015, vol. 2, pp. 631-637.

[14] Yu. Kolokolov and A. Monovskaya, "Forecasting of vernal and autumnal margins in local climate dynamics," in Proceedings of the $8^{\text {th }}$ IEEE International Conference on Intelligent Data Acquisition and Advanced Computing Systems: Technology and Applications (IDAACS'2015), Warsaw, Poland, 24-26 September 2015, vol. 2, pp. 570-575.
[15] A. Bykov and O. Kuzichkin, "Application of seismoelectric method for inspection conductive media," Applied Mechanics and Materials, vol. 490-491, pp. 1712, 2013.

[16] A. V. Ulybin and A. V. Puzanov, "About test methods of corrosion state of reinforced concrete structures," Applied Mechanics and Materials, vol. 578-579, pp. 981-986, 2014.

[17] O. R. Kuzichkin, A. A. Bykov and N. V. Dorofeev, "The resistance-acoustic method of monitoring industrial sludge plume," Applied Mechanics and Materials, vol. 770, pp. 679-685, 2015. 